IMAGING

\title{
Diffusion-weighted MRI of peripheral zone prostate cancer: comparison of tumor apparent diffusion coefficient with Gleason score and percentage of tumor on core biopsy
}

Woodfield CA, Tung GA, Grand DJ, Pezzullo JA, Machan JT, Renzulli JF 2nd

Department of Diagnostic Imaging, Rhode Island Hospital, Providence, RI, USA

AJR Am J Roentgenol. 2010; 194: W316-22.

Objective: The objective of our study was to determine the relationship between the apparent diffusion coefficient (ADC) value on diffusion-weighted imaging (DWI) and Gleason score of prostate cancer and percentage of tumor involvement on prostate core biopsy.

Materials and Methods: We performed a retrospective study of 57 patients with biopsy-proven prostate cancer who underwent endorectal MRI with DWI between July 2007 and March 2008. Regions of interest (ROIs) were drawn on ADC maps at sites of visible tumor on DW images and ADC maps. A hierarchic mixed linear model was used to compare the ADC value of prostate cancer with the Gleason score and the percentage of tumor on core biopsy.

Results: Eighty-one sites of biopsy-proven prostate cancer were visible on DW images and ADC maps. The least-squares mean ADC for disease with a Gleason score of 6 was $0.860 \times 10(-3) \mathrm{mm}(2) / \mathrm{s}$ (standard error of the mean [SEM], 0.036); Gleason score of 7, $0.702 \times 10(-3) \mathrm{mm}(2) / \mathrm{s}(\mathrm{SEM}, 0.030)$; Gleason score of 8, $0.672 \times 10(-3) \mathrm{mm}(2) / \mathrm{s}$ (SEM, 0.057); and Gleason score of 9, $0.686 \times 10(-3) \mathrm{mm}(2) / \mathrm{s}$ (SEM, 0.067). Differences between the mean ADC values for a prostate tumor with a Gleason score of 6 and one with a Gleason score of $7(\mathrm{p}=0.0096)$ and for a prostate tumor with a Gleason score of 6 and one with a Gleason score of 8 $(\mathrm{p}=0.0460)$ were significant. Comparison between the ADC and percentage of tumor on core biopsy showed a mean ADC decrease of 0.006 (range, $0.004-0.008 \times 10(-3) \mathrm{mm}(2) / \mathrm{s}$ ) for every $1 \%$ increase in tumor in the core biopsy specimen.

Conclusion: DWI may help differentiate between low-risk (Gleason score, 6) and intermediate-risk (Gleason score, 7) prostate cancer and between low-risk (Gleason score, 6) and high-risk (Gleason score > 7) prostate cancer. There is an inverse relationship between the ADC and the percentage of tumor involvement on prostate core biopsies.

\section{Editorial Comment}

In prostate cancer occurs significant reduction in the diffusion properties of water protons thus resulting in a reduction in the measured apparent diffusion coefficient (ADC) value relative to normal prostatic tissue. Several reports have been showing the utility of this technique. The combination of anatomic information obtained with conventional T2-weighted image with functional information, obtained with diffusion-weighted image, offers significant advantage over the use of either one of these techniques separately. This association significantly improves cancer detection and the accuracy in predicting the volume of cancer of the peripheral zone. This combined technique however is more effective in tumors larger than $0.5 \mathrm{~cm} 3$.

In this publication the authors show that the ADC values of prostate cancer may help differentiate between low-risk (Gleason, 6) and intermediate-risk (Gleason score 7 disease and between low-risk and high-risk (Gleason> 7). The ADC values in this study were compared with results of prostate biopsy. In other words, higher cellular density found in poorly differentiated tumors is responsible for more restricted movement of water protons and thus will present lower mean ADC values. They also showed that lower ADC values are associated with a higher percentage of cancer on core biopsy and higher Gleason score. They predicted that this feature could be useful to further direct patient treatment. 
We have to remember however that the reported sensitivity and specificity of DWI with ADC maps for detecting prostate cancer on MRI performed at $1.5 \mathrm{~T}$, range from $54 \%$ to $94 \%$ and from $61 \%$ to $100 \%$, respectively. In this study, the authors had a relatively poor detection rate, since $56 \%$ of biopsy-proven sites of prostate cancer were not visible on DWI. Sites containing tumor and visible on DWI had higher percentage of tumor on core biopsy (mean 52\%) than those not visible (mean 19\%) and higher Gleason score.

The results of this work further support our opinion that the best way to detect prostatic cancer by imaging is using a multiparametric MRI examination, which combines T2-weighted images, spectroscopy, diffusion-weighted image and dynamic contrast enhanced technique. Since each one of these techniques has inherent advantages and disadvantages, efforts have been made in order to determine which combination will present higher accuracy.

Dr. Adilson Prando

Head, Department of Radiology and Diagnostic Imaging, Vera Cruz Hospital

Campinas, São Paulo, Brazil

E-mail: adilson.prando@gmail.com

doi: 10.1590/S1677-55382010000400019

\section{Kidney and urinary tract imaging: triple-bolus multidetector CT urography as a one-stop shop- -protocol design, opacification, and image quality analysis}

Kekelidze M, Dwarkasing RS, Dijkshoorn ML, Sikorska K, Verhagen PC, Krestin GP

Department of Radiology, Erasmus Medical Center Rotterdam, Rotterdam, The Netherlands

Radiology. 2010; 255: 508-16

Purpose: To retrospectively evaluate renal, vascular, and urinary tract visualization following a single postcontrast multidetector computed tomographic (CT) urographic sequence performed with three limited-volume bolus injections.

Materials and Methods: The institutional review board approved this retrospective study. Patient informed consent was waived. Triple-bolus multidetector CT urography was performed in 110 patients. Triple-bolus protocol consisted of $30 \mathrm{~mL}$ of contrast material at $2 \mathrm{~mL} / \mathrm{sec}$ at 0 seconds, $50 \mathrm{~mL}$ at $1.5 \mathrm{~mL} / \mathrm{sec}$ at $435 \mathrm{~seconds}, 65 \mathrm{~mL}$ at $3 \mathrm{~mL} / \mathrm{sec}$ at 488 seconds, with total abdominal scanning time of 510 seconds. Two independent readers rated urinary tract opacification and qualitatively and quantitatively assessed renal parenchymal and vascular contrast enhancement. Upper urinary tract (UUT) distention was measured by one reader. Interobserver agreement was assessed by using kappa statistics.

Results: Complete opacification of the intrarenal collecting system and proximal ureter was achieved in $91 \%$ $(184$ of 202) $(\mathrm{kappa}=0.62)$ and $82 \%(166$ of 202) $(\mathrm{kappa}=0.94)$ of segments, respectively. The distal ureter was not opacified in $21 \%$ of the cases (kappa $=0.92$ ), and the bladder was not opacified in $20 \%$ of the cases. Mean distention was higher for proximal $(3.9 \mathrm{~mm})$ than for distal $(3.7 \mathrm{~mm})$ segments. Image quality of renal parenchymal enhancement was excellent in $76 \%$ of cases. Arteries showed better contrast enhancement than veins (excellent rating in $89 \%$ vs $59 \%$ of the cases). Radiation dose calculated for triple-bolus acquisition was $9.8 \mathrm{mSv}$.

Conclusion: Triple-bolus multidetector CT urography is a dose-efficient protocol acquiring corticomedullary-nephrographic-excretory and vascular enhancement phases in a single acquisition and provides sufficient opacification and distention of the UUT. Simultaneously, adequate image quality of renal parenchyma and vascular anatomy is achieved. 


\section{Editorial Comment}

Multidetector computed tomography urography (MDCTU) has become the method of choice for investigation patients with hematuria. For the adequate characterization of parenchymal, urothelial or vascular abnormalities a three-phase MDCT urographic protocols is usually necessary. With this protocol, following an unenhanced phase a single-bolus contrast material injection is made and nephrographic, and excretory phases are obtained. Using this three-phase protocol the effective radiation dose to the patient range from 15-18 $\mathrm{mSv}$. If visualization of the renal arteries and branches are necessary, an additional arterial phase is obtained, thus increasing the radiation dose to $18-20 \mathrm{mSV}$. For this reason radiologist should always perform a tailored MDCTurography protocol adequate for each patient clinical indication. The authors' presents a triple-bolus protocol designed to show all renal contrast-enhancement phases in a single acquisition. Good results were obtained with this technique, which allows the demonstration of the renal parenchyma, the renal arteries and veins and all portions of urinary tract. After an enhanced phase, a single postcontrast MDCT urographic sequence is performed with three limited-volume bolus injections. The first bolus of intravenous contrast material is for the opacification of the urinary tract, the second bolus is for the opacification of the venous system and the last bolus is performed for the opacification of the arterial system. We have found that this protocol is excellent for evaluation of potential renal donors, a characteristic group of healthy and young patients that are benefited with the use of a low-dose protocol (11-13 $\mathrm{mSv})$.

Dr. Adilson Prando

Head, Department of Radiology and Diagnostic Imaging, Vera Cruz Hospital

Campinas, São Paulo, Brazil

E-mail: adilson.prando@gmail.com

\section{PATHOLOGY}

doi: $10.1590 / S 1677-55382010000400020$

Low-grade papillary urothelial carcinoma of the urinary bladder: a clinicopathologic analysis of a post-world health organization/international society of urological pathology classification cohort from a single academic center

Miyamoto H, Brimo F, Schultz L, Ye H, Miller JS, Fajardo DA, Lee TK, Epstein JI, Netto GJ

Department of Pathology, Johns Hopkins University, Baltimore, Maryland, USA

Arch Pathol Lab Med. 2010; 134: 1160-3

Context: Few large cohort studies have addressed outcome in patients with noninvasive low-grade papillary urothelial carcinoma (LG-UrCa) following implementation of the 2004 World Health Organization/International Society of Urological Pathology (WHO/ISUP) consensus classification.

Objective: To evaluate our cohort of LG-UrCa cases classified according to 2004 WHO/ISUP to reassess outcome and interobserver agreement.

Design: Files were searched for all patients diagnosed with LG-UrCa between 1998 and 2008. All sections were reevaluated for accuracy of classification.

Results: A total of 112 cases initially diagnosed as LG-UrCa were identified. Of those, 8 of 55 cases (15\%) initially diagnosed by nonurologic pathologists were reclassified as high-grade papillary urothelial carcinoma and were excluded. The mean length of follow-up was 40.1 months (range, 2-113 months). Tumor recurrence was encountered in 56 of 104 patients (53.8\%), including 37 (35.6\%) with LG-UrCa or lower-grade tumors and 
$19(18.3 \%)$ with high-grade papillary urothelial carcinoma. Of the 19 patients demonstrating grade progression, $7(37 \%)$ also developed stage progression (invasive carcinoma, $\mathrm{n}=5$; metastatic carcinoma, $\mathrm{n}=2$ ). Seven patients eventually underwent radical cystectomy. None of the 104 patients died of bladder cancer. The mean number of recurrence episodes was 3.11. The mean durations of time to first recurrence and time to grade progression were 13.9 months and 25.1 months, respectively. The mean size of initial tumors was $1.73 \mathrm{~cm}$. There was no significant correlation between tumor size, patient age, sex, or smoking history and the likelihood for recurrence or grade progression. A significantly higher rate of recurrence was seen in patients with multiple tumors at initial diagnosis $(\mathrm{P}=.04)$.

Conclusions: A tendency to underdiagnose high-grade papillary urothelial carcinoma continues to exist. More than half $(53.8 \%)$ of patients with LG-UrCa developed recurrence, with an $18.3 \%$ incidence of grade progression and a $6.7 \%$ incidence of stage progression. Patients with multiple initial tumors had significantly higher risk of developing recurrence.

\section{Editorial Comment}

This is a large cohort study of outcome of patients with noninvasive low-grade urothelial carcinoma using the World Health Organization/International Society of Urological Pathology (WHO/SIPU) consensus classification. From a total of 104 patients, 53.8\% developed recurrence, with an $18.3 \%$ incidence of grade progression and a $6.7 \%$ incidence of stage progression. Patients with multiple initial tumors had significantly higher risk of developing recurrence.

The World Health Organization/International Society of Urological Pathology (WHO/SIPU) consensus classification was held in Boston in 1998 during the United States and Canadian Academy of Pathology (USCAP) meeting. The results were published in the American Journal of Surgical Pathology (1). The most important recommendations of the meeting were:

1. It was recommended not to use the traditional term "dysplasia". For the term moderate dysplasia was recommended to use low-grade intra-urothelial neoplasia and for severe dysplasia or flat carcinoma in situ the term high-grade intra-urothelial neoplasia. Cases with slight dysplasia should be reported by the pathologists.

2. The 1, 2 and 3 grading by the World Health Organization was replaced by low-grade or refer to grade 1, and high-grade to refer to grades 2 or 3.

3. "Urothelial papilloma" without qualifiers refers to the exophytic variant of papilloma, defined as a discrete papillary growth with a central fibrovascular core lined by urothelium of normal thickness and cytology. It is a rare benign condition typically occurring as a small, isolated growth commonly, but not exclusively, in younger patients.

4. It was introduced the term "papillary urothelial neoplasm of low malignant potential". This is a papillary urothelial lesion with an orderly arrangement of cells within papillae with minimal architectural abnormalities and minimal nuclear atypia irrespective of cell thickness. Patients with these tumors are at risk of developing new bladder tumors of a similar histology. However, occasionally these subsequent lesions manifest as urothelial carcinoma, such that follow-up of the patient is warranted. In the standard classification this tumor corresponds to the "papillary urothelial carcinoma, grade 1 (low-grade), pTa". This new category avoid labeling a patient as having cancer, which has psychosocial and financial (e.g. insurance) implications, but neither is a benign lesion (e.g., papilloma) diagnosed, so the patient might be adequately followed (2).

\section{References}

1. Epstein JI, Amin MB, Reuter VR, Mostofi FK: The World Health Organization/International Society of Urological Pathology consensus classification of urothelial (transitional cell) neoplasms of the urinary bladder. Bladder Consensus Conference Committee. Am J Surg Pathol. 1998; 22: 1435-48. 


\section{Urological Survey}

2. Epstein JI, Reuter VE, Amin MB: Biopsy Interpretation of the Bladder, 2nd ed. Philadelphia, Lippincott Williams \& Wilkins. 2010.

Dr. Athanase Billis

Full-Professor of Pathology

State University of Campinas, Unicamp

Campinas, São Paulo, Brazil

E-mail:athanase@fcm.unicamp.br

doi: 10.1590/S1677-55382010000400021

\section{Intensity of stromal changes predicts biochemical recurrence-free survival in prostatic carcinoma}

Tomas D, Spajić B, Milošević M, Demirović A, Marušić Z, Krušlin B

Department of Pathology

Scand J Urol Nephrol. 2010; 12 [Epub ahead of print]

Abstract: The reactive stroma of prostate cancer contains a mixture of myofibroblasts and fibroblasts, while fully differentiated smooth-muscle cells are very rare or absent. In experimental prostate cancer models, prostatic stromal cells promote angiogenesis and stimulate prostate tumorigenesis. The aim of this study is to analyse whether the intensity of stromal changes can predict survival in patients with prostatic carcinoma. Material and methods: Stromal reaction was quantified histochemically and imunohistochemically in 50 patients treated with radical prostatectomy for clinically localized prostate carcinoma and its relationship with established prognostic factors was assessed.

Results: Kaplan-Meier analysis showed a significant association between the pattern of vimentin and desmin expression and the length of disease-free period; patients with a higher vimentin or lower desmin expression had a shorter disease-free period. On multivariate analysis only vimentin expression (odds ratio 4.06, 95\% confidence interval 1.01-16.26, $\mathrm{p}=0.049$ ) was a significant predictor of biochemical recurrence. In patients with identical Gleason pattern and Gleason score the level of vimentin expression could identify patients with a higher risk of disease recurrence.

Conclusions: Intensity of stromal changes could serve as an independent prognostic factor in the assessment of biochemical recurrence-free survival. Among prostate cancer patients with an identical Gleason score, it could identify patients with a higher risk of biochemical recurrence. Thus, stromal changes and their intensity could serve as a novel marker for the recognition of patients with an increased risk of disease recurrence.

\section{Editorial Comment}

There is evidence that prostate carcinogenesis is influenced and controlled by cellular interactions derived from a complex relationship between stromal, epithelial and extracellular matrix components. In prostate cancer as well as in many other cancers, the stromal microenvironment is different from the corresponding normal stroma. There is increased microvessel density, inflammatory cells and modified fibroblasts. The latter are called myofibloblasts or cancer-associated fibroblasts and are considered to play a central role in the complex process of tumor-stroma interaction and consequently in the tumor growth, spread and metastasis, and could also represent an important target for cancer therapies.

The study by Tomas' et al. showed that the intensity of stromal changes could serve as an independent prognostic factor in the assessment of biochemical recurrence-free survival in patients submitted to radical prostatectomy. The only other group studying the predictive value of reactive stroma (desmoplasia) for biochemical 
recurrence following surgery is from Baylor College in Houston, Texas. Yanagisawa N et al. (1) have shown that reactive stromal grading in biopsies was correlated with adverse pathological parameters in the prostatectomy and independent predictor of biochemical recurrence. The authors concluded that quantitation of reactive stroma and recognition of the desmoplastic cancer in $\mathrm{H} \&$ E-stained biopsies is useful to predict biochemical recurrence in prostate carcinoma patients independent of Gleason grade and prostate-specific antigen. Obviously more studies are needed for definitive conclusions.

\section{Reference}

1. Yanagisawa N, Li R, Rowley D, Liu H, Kadmon D, Miles BJ, et al.: Stromogenic prostatic carcinoma pattern (carcinomas with reactive stromal grade 3) in needle biopsies predicts biochemical recurrence-free survival in patients after radical prostatectomy. Hum Pathol. 2007; 38: 1611-20.

Dr. Athanase Billis

Full-Professor of Pathology

State University of Campinas, Unicamp

Campinas, São Paulo, Brazil

E-mail: athanase@fcm.unicamp.br

\section{RECONSTRUCTIVE UROLOGY}

doi: $10.1590 / \mathrm{S} 1677-55382010000400022$

\section{Internal urethrotomy and intraurethral submucosal injection of triamcinolone in short bulbar urethral strictures}

Mazdak H, Izadpanahi MH, Ghalamkari A, Kabiri M, Khorrami MH, Nouri-Mahdavi K, Alizadeh F, Zargham M, Tadayyon F, Mohammadi A, Yazdani M

Al-Zahra Hospital, Isfahan University of Medical Sciences, Isfahan, Iran

Int Urol Nephrol. 2009; 1. [Epub ahead of print]

Objectives: In clinical practice, internal urethrotomy is an easy procedure and is offered as a first modality for treatment of short urethral strictures. Internal urethrotomy refers to any procedure that opens the stricture by incising or ablating it transurethrally. The most common complication of internal urethrotomy is stricture recurrence. The curative success rate of internal urethrotomy is approximately $20 \%$. Triamcinolone has antifibroblast and anticollagen properties. This study evaluated the efficacy of triamcinolone in the prevention of anterior urethral stricture recurrence after internal urethrotomy.

Methods: Fifty male patients with anterior urethral stricture were randomized to undergo internal urethrotomy with or without urethral submucosal injection of triamcinolone. Using general anesthesia urethrotomy was performed. Triamcinolone ( $40 \mathrm{mg}$ ) was injected submucosally at the urethrotomy site in 25 patients. The patients were followed for at least 12 months and the stricture recurrence rate was compared between the two groups.

Results: 23 patients in the triamcinolone group and 22 in the control group completed the study. There were no significant differences in the baseline characteristics of the patients or the etiology of the stricture between the two groups. Mean follow-up time was $13.7 \pm 5.5$ months (range: 1-25 months). Urethral stricture recurred in five patients $(21.7 \%)$ in the triamcinolone group and in 11 patients $(50 \%)$ in the control group $(\mathrm{P}=0.04)$. Conclusions: Injection of triamcinolone significantly reduced stricture recurrence after internal urethrotomy. Further investigations are warranted to confirm its efficacy and safety. 


\title{
Editorial Comment
}

In this study men were randomized to steroid injection vs. no injection after internal urethrotomy for short $(<1.5 \mathrm{~cm})$, treatment-naïve bulbar urethral strictures. Follow-up was with urethrography and urethroscopy every 6 months or when symptoms recurred. Treatment failure was defined as need for repeat treatment. Patients were similar in the two groups in all respects. Recurrence rate was $22 \%$ in the treatment group and $50 \%$ in the control group at mean follow-up of just over a year. These results are encouraging and we look forward to future reports with longer follow-up. Some shortcomings of this study deserve mention and point to areas for improvement in the design of future studies which attempt to answer this question. The study was not blinded and there was no placebo. Future studies should blind the surgeon and patient to injection with steroid vs. saline. The study outcome is fairly subjective. A more objective outcome would be a standardized assessment such as the ability to pass a cystoscope.

\author{
Dr. Sean P. Elliott \\ Department of Urology Surgery \\ University of Minnesota \\ Minneapolis, Minnesota, USA \\ E-mail: selliott@umn.edu
}

doi: $10.1590 / S 1677-55382010000400023$

\section{Antegrade endourethroplasty with free skin graft for recurrent vesicourethral anastomotic strictures after radical prostatectomy}

Kuyumcuoglu U, Eryildirim B, Tarhan F, Faydaci G, Ozgül A, Erbay E

Dr. Lütfi Kirdar Kartal Training and Research Hospital, Istanbul, Turkey

J Endourol. 2010; 24: 63-7

Purpose: To investigate the efficacy of the antegrade endourethroplasty technique for the management of frequently recurrent vesicourethral anastomotic strictures that develop after retropubic radical prostatectomy.

Patients and Methods: Between January 2006 and February 2008, endoscopic antegrade urethroplasty was performed in 11 patients with recurrent vesicourethral anastomotic strictures that developed after retropubic radical prostatectomy (RRP). The mean age of the patients was 64.6 years. In the first step of this two-step procedure, the graft bed was prepared by transurethral resection of the vesicourethral anastomotic stricture region. In the next step, after 3 days, an Amplatz sheath was placed in the urinary bladder suprapubically. Then, an endobronchial catheter was inserted from the external urethral meatus and extended out of the body from the suprapubic region through the Amplatz sheath. A graft taken from anteromedial section of the arm was tubularized on the catheter balloon. The graft was placed into the bladder neck antegradely under endoscopic vision. Subsequently, the graft carrier catheter was fixed by previously placed two polypropylene sutures inserted into the proximal and distal part of the stricture zone percutaneously from the perineum. The transurethral catheter was taken out delicately on postoperative day 21.

Results: Urethral patency succeeded in 6 of the 11 (54.5\%) patients, and maximum flow rate was more than $13 \mathrm{~mL} / \mathrm{s}$ in follow-up. Graft necrosis occurred in two patients, and the stricture recurred in three patients in two months postoperatively.

Conclusion: Antegrade endourethroplasty may be a suitable alternative to open surgical reconstruction in selected patients with recurrent bladder neck stricture following RRP. Further studies, including more patients with modifications, are needed to improve the success rate. 


\section{Editorial Comment}

A minimally-invasive approach is appropriate in surgery when it offers results that are similar to those obtained with an open approach but with less morbidity. For this reason, the recalcitrant bladder neck contracture is the ideal stricture site for the development of the endoscopic urethroplasty. The alternatives are either unsuccessful (repeat dilation or urethrotomy) or are associated with high morbidity (urethral stent or open reconstruction). Still, concerns remain with this approach. First, a successful graft requires a healthy graft bed. Certainly, the recently resected and fulgurated TUR area is not an ideal graft bed. Second, results with tubular grafts or flaps in open urethral reconstruction have been poor. It is unclear why they should be any better with an endoscopic approach. Still, these initial results are encouraging and given the alternatives, a 55\% success rate and flow rates of only $13-18 \mathrm{cc} / \mathrm{s}$ are pretty good in these complex patients with few alternatives.

Dr. Sean P. Elliott

Department of Urology Surgery

University of Minnesota

Minneapolis, Minnesota, USA

E-mail:selliott@umn.edu

\section{UROLOGICAL ONCOLOGY}

doi: $10.1590 / S 1677-55382010000400024$

Long-term efficacy results of EORTC Genito-Urinary Group randomized phase 3 study 30911 comparing intravesical instillations of epirubicin, bacillus Calmette-Guérin, and bacillus Calmette-Guérin plus isoniazid in patients with intermediate- and high-risk stage Ta T1 urothelial carcinoma of the bladder

Sylvester RJ, Brausi MA, Kirkels WJ, Hoeltl W, Calais Da Silva F, Powell PH, Prescott S, Kirkali Z, van de Beek C, Gorlia T, de Reijke TM; EORTC Genito-Urinary Tract Cancer Group

EORTC Headquarters, Brussels, Belgium

Eur. Urol. 2010; 57: 766-773

Background: Intravesical chemotherapy and bacillus Calmette-Guérin (BCG) reduce the recurrence rate in patients with stage Ta T1 urothelial bladder cancer; however, the benefit of BCG relative to chemotherapy for long-term end points is controversial, especially in intermediate-risk patients.

Objective: The aim of the study was to compare the long-term efficacy of BCG and epirubicin.

Design, Setting, and Participants: From January 1992 to February 1997, 957 patients with intermediate- or high-risk stage Ta T1 urothelial bladder cancer were randomized after transurethral resection to one of three treatment groups in the European Organization for Research and Treatment of Cancer Genito-Urinary Group phase 3 trial 30911.

Intervention: Patients received six weekly instillations of epirubicin, BCG, or BCG plus isoniazid (INH) followed by three weekly maintenance instillations at months $3,6,12,18,24,30$, and 36 .

Measurements: End points were time to recurrence, progression, distant metastases, overall survival, and disease-specific survival.

Results and Limitations: With 837 eligible patients and a median follow-up of $9.2 \mathrm{yr}$, time to first recurrence $(\mathrm{p}<0.001)$, distant metastases $(\mathrm{p}=0.046)$, overall survival $(\mathrm{p}=0.023)$, and disease-specific survival $(\mathrm{p}=0.026)$ were significantly longer in the two BCG arms combined as compared with epirubicin; however, there was no difference for progression. Three hundred twenty-three patients with stage T1 or grade 3 tumors were high risk, 
and the remaining 497 patients were intermediate risk. The observed treatment benefit was at least as large, if not larger, in the intermediate-risk patients compared with the high-risk patients.

Conclusions: In patients with intermediate- and high-risk stage Ta and T1 urothelial bladder cancer, intravesical BCG with or without INH is superior to intravesical epirubicin not only for time to first recurrence but also for time to distant metastases, overall survival, and disease-specific survival. The benefit of BCG is not limited to just high-risk patients; intermediate-risk patients also benefit from BCG.

\section{Editorial Comment}

The efficacy of BCG against recurrences in high-risk patients has long been recognized. Previous papers and meta-analyses have also shown that BCG favorably acts against progression of disease. Even further data supported the notion that this was also true for intermediate-risk patients. However, many authors were not satisfied with the database for these conclusions and in recent times, others have contributed with opposite results.

This EORTC paper now, with its high number of patients ( 975 randomized and 837 eligible) and its inherent quality of statistical analysis puts an end to many of these questions (and poses several new ones). In this randomized study of BCG against epirubicin (EPI) the results clearly show an advantage of BCG in terms of time to first recurrence, and even more important, in terms of distant metastases and survival (!). Moreover, the favorable results were even more pronounced in the intermediate-risk group. The question still remaining is why time to progression was not different between the treatment groups. According to the authors, BCG appeared to reduce the risk of progression (hazard ratio 0.56$)$ but there were too few progressions $(n=25)$ to make meaningful comparisons.

Certainly, this paper will have a significant impact on the guidelines on non-muscle invasive bladder cancer and on the routine clinical treatment of this disease.

Dr. Andreas Bohle

Professor of Urology

HELIOS Agnes Karll Hospital

Bad Schwartau, Germany

E-mail: boehle@urologie-bad-schwartau.de

doi: $10.1590 / S 1677-55382010000400025$

\section{Detrusor muscle in the first, apparently complete transurethral resection of bladder tumour specimen is a surrogate marker of resection quality, predicts risk of early recurrence, and is dependent on operator experience}

Mariappan P, Zachou A, Grigor KM; for the Edinburgh Uro-Oncology Group

Department of Urology, Western General Hospital, Edinburgh, United Kingdom

Eur. Urol. 2010; 57: 843-849

Background: An European Organisation for Research and Treatment of Cancer analysis of multicentre trials found significant interinstitutional variability in recurrence rates at first follow-up cystoscopy (RR-FFC) and attributed this to variable transurethral resection of bladder tumour (TURBT) quality.

Objective: To determine whether resection of detrusor muscle (DM) in the first, apparently complete TURBT is a surrogate marker of quality and whether the presence of DM is dependent on a surgeon's experience.

Design, Setting, and Participants: Over a 2-yr period, patients with new bladder tumours that were judged to have been completely resected were recruited from our prospectively maintained bladder tumour database. Strict exclusion criteria were applied. 
Measurements: Prospectively recorded tumour size, tumour multiplicity, surgeon category, DM status, grade and stage of tumour, and findings at first follow-up cystoscopy (at $3 \mathrm{mo}$ ) and at early re-TURBT were evaluated. Surgeons were stratified into seniors (consultants and year 5 or year 6 trainees) and juniors (trainees lower than year 5). Early recurrence (for calculating RR-FFC) was defined as pathologically confirmed tumour on early re-TURBT or recurrence at the first follow-up cystoscopy. Logistic regression multivariate analyses were carried out to determine associations between variables.

Results and Limitations: In a total of 356 patients, DM was present in 241 patients $(67.7 \%)$. Multivariate analyses revealed that large tumours, high-grade tumours, and surgery by senior surgeons was independently associated with the presence of DM in the resected specimens. The RR-FFCs when DM was absent and present were $44.4 \%$ and $21.7 \%$, respectively (odds ratio: $2.9 ; 95 \%$ confidence interval: $1.6-5.4 ; \mathrm{p}=0.0002$ ). The absence of DM and resection by less experienced surgeons independently predicted a higher RR-FFC. This association was also seen in small and low-grade tumours. The number of patients in this study appears modest, and further validation may be required.

Conclusions: DM absence or presence in the first, apparently complete TURBT specimen appears to be a surrogate marker of resection quality by independently predicting the RR-FFC, which is also dependent on surgeon experience.

\title{
Editorial Comment
}

The quality of surgery is an important fact. This retrospective analysis of resection quality and analysis of early tumor recurrences now gives some hard arguments in favor of a thorough and radical, deep transurethral resection including detrusor muscle (DM). Through all groups analyzed senior surgeons had better results than junior surgeons in terms of detrusor muscle included in specimen. The important fact is that this directly translated into early tumor recurrence at three months. The absence of DM was associated with a significantly higher risk of both early recurrence at first follow-up cystoscopy and residual disease at early re-TURBT. In patients with TaG1 and TaG2 tumors, the risk of early recurrence was $34.5 \%$ in the absence of DM, compared to $14.5 \%$ when DM was present $(\mathrm{p}=.005)$. In patients with G3 tumors, the overall risk of recurrence was 5 -fold higher when DM was absent $(<0.001)$. In patients with $\mathrm{T} 1$ disease the recurrence rates were $81.3 \%$ and $34.9 \%$ when DM was absent or present, respectively $(\mathrm{p}=.002)$.

Therefore, do a good job and mind the presence of detrusor muscle in your TURBT specimen!

\author{
Dr. Andreas Bohle \\ Professor of Urology \\ HELIOS Agnes Karll Hospital \\ Bad Schwartau, Germany \\ E-mail: boehle@urologie-bad-schwartau.de
}

NEUROLOGY \& FEMALE UROLOGY

doi: 10.1590/S1677-55382010000400026

\section{Urethral diverticula in women: discrepancies between magnetic resonance imaging and surgical findings}

Chung DE, Purohit RS, Girshman J, Blaivas JG

Department of Urology, Weill Medical College of Cornell University, New York, NY, USA

J Urol. 2010; 183: 2265-9 
Purpose: Some groups consider magnetic resonance imaging the gold standard to diagnose urethral diverticula with up to $100 \%$ reported sensitivity. We describe cases contradicting this paradigm and identify reasons for discrepancies.

Materials and Methods: We searched a database for women who underwent urethral diverticulum surgery from 1998 to 2008 and also underwent preoperative magnetic resonance imaging. Images were reviewed by a blinded panel of urologists and a radiologist. They came to consensus on the presence or absence, site and anatomy of urethral diverticulum or cancer, and compared operative findings. Discrepancies were classified as errors in urethral diverticulum or cancer diagnosis and errors in urethral diverticulum anatomy or site.

Results: Of 76 patients who underwent diverticulectomy 41 also underwent magnetic resonance imaging, of whom $10(24.4 \%)$ had a discrepancy between magnetic resonance imaging and surgical findings. In 6 of these cases there were diagnosis errors and diverticula were not seen on magnetic resonance imaging in 3. One urethral diverticulum each was misdiagnosed as Bartholin's cyst and as a typical post-collagen injection appearance. A sterile abscess was incorrectly diagnosed as a urethral diverticulum. In 2 patients magnetic resonance imaging did not detect cancer within the diverticulum. A major discrepancy in anatomy made intraoperative decision making difficult in 2 patients.

Conclusions: In cases clinically suspicious for urethral diverticulum magnetic resonance imaging had a $24.4 \%$ error rate. Serious consequences are failure to detect cancer and suboptimal treatment for urethral diverticulum. The reason for the high magnetic resonance imaging accuracy rate in other series may be that in the absence of radiological confirmation some surgeons may choose not to perform surgery. Magnetic resonance imaging is useful to assess urethral diverticula but physicians should be aware of its limitations.

\section{Editorial Comment}

A thoughtful presentation questioning the acceptance of MRI of the urethra as the absolute sensitive and specific test to identify, localize, and characterize urethral diverticula. The authors found an approximate $25 \%$ rate of diagnostic discrepancy or misdiagnosis in those patients who had undergone MRI for the diverticulum. Of keen interest was that almost $10 \%$ of the patients ultimately found to have a diverticulum were noted to have a negative MRI. The authors provide an excellent discussion reviewing their thoughts on why the MRI may fail to either identify or properly characterize a urethral diverticulum.

A good take home message after reading this work is that when evaluating for a urethral diverticulum, one should not abandon clinical judgment and suspicion or forget historical studies such as the double balloon retrograde urethrogram in the face of a negative MRI.

Dr. Steven P. Petrou

Professor of Urology, Associate Dean

Mayo School of Graduate Medical Education

Jacksonville, Florida, USA

E-mail:petrou.steven@mayo.edu

doi: 10.1590/\$1677-55382010000400027

\section{Requiem for the suburethral tape}

Fletcher SG, Zimmern PE

Female Pelvic Medicine, Reconstructive Surgery and Neurourology, UT South-Western Medical Center, Dallas, TX, USA

BJU Int. 2010; 105: 445-8 
In this article, the author discusses the treatment options for women with bothersome stress urinary incontinence (SUI). He comments on the strong connection between the urethra and anterior vaginal wall and the complications related to the synthetic suburethral tapes. Further, the use of a vaginal wall support procedure with a pubovaginal sling and a mesh in the vaginal wall to treat UI are discussed.

\title{
Editorial Comment
}

An intellectual commentary discussing suburethral tape procedures, the potential reasons for their success as well as their complications. The authors note that based on the integral theory, the suburethral sling will help restore "deficient pubo-urethral ligaments to their normal anatomy (sic)". They note that in their personal experience they have not identified a distinct pubourethral ligament. This observation has been discussed in previous editorials in this journal (1) and may explain the ability to maintain urinary continence after a suprameatal transvaginal urethrolysis (2). In addition, the article does quote a very candid if not surprising lower level of success with suburethral sling placement based on their Level 1 evidence.

This review is a worthwhile read for the surgeon interested in expanding both central and peripheral understanding of sub midurethral sling surgery.

\section{References}

1. Petrou SP: Editorial Comment: What are the supportive structures of the female urethra? Int Braz J Urol. 2006; 32 : 249-50.

2. Petrou SP, Brown JA, Blaivas JG: Suprameatal transvaginal urethrolysis. J Urol. 1999; 161: 1268-71.

\author{
Dr. Steven P. Petrou \\ Professor of Urology, Associate Dean \\ Mayo School of Graduate Medical Education \\ Jacksonville, Florida, USA \\ E-mail: petrou.steven@mayo.edu
}

\section{PEDIATRIC UROLOGY}

doi: $10.1590 / S 1677-55382010000400028$

Infant communicating hydroceles -- do they need immediate repair or might some clinically resolve?

Koski ME, Makari JH, Adams MC, Thomas JC, Clark PE, Pope JC 4th, Brock JW 3rd

Department of Urology, Division of Pediatric Urology, Monroe Carell Jr Children's Hospital at Vanderbilt, Vanderbilt University Medical Center, Nashville, TN, USA

J Pediatr Surg. 2010; 45: 590-3

Purpose: Infant hydroceles that are communicating by history (fluctuation in size) or examination (reducible fluid) are often repaired soon after presentation. We have followed a series of infant boys with such hydroceles and reviewed their early natural history.

Materials and Methods: Since 1998, we have followed 174 infant boys presenting with an apparent communicating hydrocele without immediate surgical repair. All boys were initially seen before 18 months of age and most (168) by 12 months. Most had been full term at delivery, although 32 had been premature ( $<37$ weeks' gestational age) and 11 extremely so $(<32$ weeks). Most boys (120) had bilateral hydroceles at presentation. Results: Of the 110 boys followed to disposition, 69 (62.7\%) had complete resolution without surgery by a mean age of 11.7 months. Forty-one patients (37.3\%) underwent surgery for correction at a mean age of 14 months 
because of persistence in size or development of a hernia. Six developed a hernia during observation, none of whom had any episode of incarceration. Only 2 patients with apparent resolution subsequently had recurrence with a hernia. Age at presentation and gestational age at birth showed no effect on resolution. The hydroceles of 64 boys had improved in size after a mean follow-up of 13.9 months when last seen.

Conclusions: Many infant hydroceles that are communicating by history or examination do resolve clinically without surgery and deserve observation. Progression to hernia was rare in our experience and did not result in incarceration. Consequently, little risk is taken by initial observation.

\title{
Editorial Comment
}

The authors of this retrospective study propose that observation of communicating hydroceles in young infants is warranted given a fairly high resolution rate and low rate of progression to a true hernia in their series. In addition, there were no episodes of incarceration of these hernias. Because the natural tendency of most pediatric urologists and pediatric surgeons is to repair communicating hydroceles near the time of presentation, we have previously had little data to demonstrate the natural history of these patients. This series provides nice data for us and suggests that observation may be reasonable for many of these patients. I suspect that over a 9-year period of time, there were more than 174 patients younger than 18 months who presented to their institution with a communicating hydrocele. It would be interesting to know what criteria were used to determine which patients should be followed and which patients should be repaired without observation.

\author{
M. Chad Wallis \\ Division of Pediatric Urology \\ University of Utah \\ Salt Lake City, Utah, USA \\ E-mail: chad.wallis@hsc.utah.edu
}

doi: 10.1590/S1677-55382010000400029

\author{
Later toilet training is associated with urge incontinence in children \\ Barone JG, Jasutkar N, Schneider D \\ Division of Urology, Section of Pediatric Urology, Robert Wood Johnson Medical School, New Brunswick, NJ, \\ USA \\ J Pediatr Urol. 2009; 5: 458-61
}

Objective: The objective of this study was to determine if later toilet training is associated with urge incontinence in children.

Methods: We used a case-control study design to yield level 2 evidence.

Results: Initiation of toilet training after 32 months of age was associated with urge incontinence $(\mathrm{P}=0.02)$.

Conclusion: For children who display signs of toilet-training readiness, training should be initiated prior to 32 months of age to reduce the risk for urge incontinence.

\section{Editorial Comment}

This was a case controlled study matching 58 patients who presented to a pediatric urology office with urge incontinence and 157 controls from a general pediatric practice. The patient ages range between 4 and 12 years. Parents were given a questionnaire that included demographics, socioeconomic status, urinary symptoms, and age at the initiation of toilet training. Parents also indicated whether they used a child or parent-oriented approach. The investigators found that the only statistically significant difference between cases and controls 
was the mean age at toilet training. Patients with urge incontinence were trained at a mean age of almost 32 months whereas the control group was trained at just under 29 months. There was no difference in the type of toilet training method utilized.

Parents are often interested in getting advice on when and how to potty train their children. The study shows an association between urge incontinence and potty training at a later age. The authors point out that there is data to suggest that potty training at an age less than 27 months might not be helpful either. Certainly, each child and family must be looked at individually; however, we now have more evidence to suggest that there may be an "ideal time" to initiate potty training.

M. Chad Wallis

Division of Pediatric Urology

University of Utah

Salt Lake City, Utah, USA

E-mail: chad.wallis@hsc.utah.edu 